\title{
Pathogen Load and the Nature of Ingroup Derogation: Evolution of Ingroup Derogation Based on the Sexual Penna Model
}

\author{
Chun $\mathrm{Hu}$ \\ Cognition and Human Behavior Key Laboratory of Hunan \\ Province \\ Hunan Normal University \\ Changsha, China \\ c_lakes@163.com
}

\author{
Xiongfeng Li \\ Cognition and Human Behavior Key Laboratory of Hunan \\ Province \\ Hunan Normal University \\ Changsha, China \\ 543269224@qq.com
}

\author{
Qi Wu* (Corresponding author) \\ Cognition and Human Behavior Key Laboratory of Hunan \\ Province \\ Hunan Normal University \\ Changsha, China \\ sandwich624@yeah.net \\ Chun Hu and Xiongfeng Li contributed equally to this work
}

\begin{abstract}
Ingroup derogation is a preference and affinity for outgroup members over ingroup members which contradicts both expert and lay beliefs. In present study, we further tested the evolutionary ingroup derogation hypothesis by using computational modeling analysis based on the modified sexual Penna model to simulate the evolution of ingroup derogation in conditions that both the ingroup and outgroup members can incur disease threats. The results showed that the difference of pathogen loads between two different regions would affect the intergroup attitudes of the social groups within these two regions. That is, the social group in the area with relative lower prevalence of disease would display the ingroup favoritism attitude, while the group in the area with relatively higher prevalence of disease would evolve the ingroup derogation attitude. In addition, the results also showed that the strength of the ingroup derogation attitude was positively correlated with this difference. These results indicate that ingroup derogation could be evolved under a broad range of conditions which are easily to be satisfied in the real world and suggest that ingroup derogation is a special adaptation to a particular situation in which ingroup members pose more threat of diseases than outgroup members.
\end{abstract}

Keywords-ingroup derogation; sexual Penna model; computer simulation; disease threat

\section{INTRODUCTION}

Ingroup derogation, also called outgroup favoritism, is a preference for outgroup members over ingroup members [1]. It's very similar but contrary to the ubiquitous ingroup bias which is an affinity for ingroup members over outgroup members [2]. Previous studies have found that ingroup derogation could be observed among specific group members,

This work was supported by the National Natural Science Foundation of China (grant number 31300870) and the Hunan Normal University (grant number 13XQN01\&2015y x08). such as in small groups with lower group status [3, 4]. For example, African

Americans have been found to consent to the stereotypes about themselves [3]. Researchers also discovered the "black sheep effect" that people will be more willing to be distanced from the deviant ingroup members whose norms or values are very different from others [4]. In addition, the studies found that ingroup derogation seems to be particularly prevalent in Eastern cultures [5]. For example, researchers have found that the mainland Chinese rated the faces and names of outgroup members as more beautiful and better than their ingroup counterparts [6]. Ma-Kellams et al. also reported that the East Asians implicitly associated outgroup members with more positive traits than their own ingroup members [5, 7].

The existence of ingroup derogation is a paradox in an evolutionary sense. Numerous studies have indicated that natural selection should favor the individuals who prefer their ingroups and eliminate the individuals who prefer outgroups, since outgroups bring greater threats of disease and the ingroups are much safer [8-13]. Therefore, from the evolutionary perspective, favoritism toward ingroup members is an adaptation, and favoritism toward outgroup members is not viewed as adaptations but should be viewed as maladaptation, which makes it difficult to explain the prevalence and persistence of ingroup derogation phenomenon.

In recent years, an evolutionary hypothesis was proposed to explain the function of ingroup derogation attitudes found among East Asian participants. It was proposed that ingroup derogation is a special adaptation to a particular situation in which ingroup members pose more threat of diseases than outgroup members. That is, if there are outbreaks of some 
emerging diseases in the local habitat of ingroup members, or somehow the pathogen load within the local habitat of ingroup members become much higher than the pathogen load within the local habitat of outgroup members (such as environmental change), it would be much more easier to catch an infectious disease via an ingroup member than via an outgroup member [14]. Under such circumstances, it would be more adaptive to derogate and avoid ingroup members than to bond with them. Thus, this hypothesis suggests that the East Asian participants derogate their ingroups because they are respond to heuristic cues indicating the disease threat incurred by the ingroups has become stronger than the disease threat incurred by the outgroups [14].

Evidence supporting this hypothesis has been obtained. For example, studies have shown that the disease load in China has always been higher than in Europe by both historical and contemporary measures [15], and China is the main place in which the phenomenon of ingroup derogation has been discovered [5, 6]. In addition, researchers have also found that heightened disease concerns would lead the Chinese participants to display stronger ingroup derogation attitudes [14]. Although the researchers have found the relationship between infectious disease and ingroup derogation attitudes through behavioral experiments [14], such a hypothesis is still speculative. No direct evidence showed that such an evolutionary process exist in human history. In fact, in the absence of a time machine or any clear fossil record about social interactions, it is impossible for us to draw completely confident conclusions about this hypothesis. An approximate substitution is to utilize computational models to infer the historical process of human evolutionary history [16].

The sexual Penna model is an agent-based model which has been widely accepted and applied in the field of biological evolution modeling due to its predictability and simplicity [17]. Researchers have employed it in many biological and ecological studies, such as, the phenomenon of sympatric speciation [18], the spreading of epidemic [19], the evolution of intelligence [20], and so on.

Since its success and validity in evolution modeling, in present study, we used sexual Penna model to simulate the historical process of behavioral mechanism evolution and set different levels of disease load in different groups to examine necessary conditions for the evolution of ingroup derogation mechanism.

\section{The MODIFIED SEXUAL PENNA MODEL}

\section{A. Agents and Their Habitat}

The sexual Penna model describes the evolution process in terms of agents. In our model, all populations are divided into two social group $A$ and $B$, the initialization number of agents in each of these two group is respectively represented by $N_{a}$ and $N_{b}$, and each group has equal living space which is represented by a lattice, where group $A$ lives in $L_{A}$ and group $B$ lives in $L_{B}$. The maximum capacity of each lattice is respectively represented by $N A_{\max }$ and $N B_{\max }$. Different regions can carry different kinds of infectious pathogens (such as bacteria, parasites, viruses, etc.) [21]. Due to the adaption of the pathogens to the local environment [21], the $L_{A}$ only hosts one pathogen $a$, while the $L_{B}$ hosts another pathogen $b$. These two pathogens are represented by Bit-string $i l l_{a}$ and Bit-string $i l l_{b}$ with $A_{\max }$ in length (see Fig. 1). The disease load factors of group $A$ and $B$ are separately represented as $F_{a}$ and $F_{b}$, the disease load determines the prevalence of the disease. The higher the disease load, the higher the value of the prevalence.

The genes of agents are represented by three pairs of Bitstrings (also called chromosomes in this model) with a length of $A_{\max }$ (see Fig. 1). The contents of these Bit-strings are fixed at the time of the agent's birth. The Health Bit-string controls the healthy status and the immune process of the agent. With the agent's growth, the Health Bit-string will open the corresponding location of the gene pair. Such as, at the position of $i$, its expression is $H(i)=H_{1}(i) \wedge H_{2}(i), H_{1}(i)$ and $H_{2}(i)$ are gene pairs of Health Bit-string, and The symbol ' $\wedge$ ' indicates logical meaning of 'and'. If the value of $H(i)$ in this year is 1 , the agent will be affected by a genetic disease. And if the number of genetic diseases attacking the agent reaches the threshold $T$, the agent will die. Due to the restriction of food and space, the agents also die with a certain probability, for the agent in group $A$, its expression is $V_{a}=N_{t}(a) / N A_{\text {max }}$; As the same, for the agent in group $B$, its expression is $V_{b}=$ $N_{t}(b) / N B_{\max }$, where $N_{t}(a)$ and $N_{t}(b)$ is the number of agents in group $A$ and group $B$ at time of $t$, respectively.

The Ingroup Bit-string controls the agent's attitude for ingroup members. Its expression is $F_{i n}=\sum_{i-1}^{A_{\max }} I(i) / A_{\max }$, where $I(i)=I_{1}(i) \wedge I_{2}(i), I_{1}(i)$ and $I_{2}(i)$ are gene pairs of Ingroup Bit-string at position $i$, respectively. The same principle also applies to the outgroup attitudes in which $F_{\text {out }}=\sum_{i-1}^{A_{\max }} O(i) / A_{\max }$, where $O(i)=O_{1}(i) \wedge O_{2}(i), O_{1}(i)$ and $\mathrm{O}_{2}(i)$ are gene pairs of Outgroup Bit-String at position $i$, respectively. The ingroup and outgroup Bit-string determine the preference of a specific agent for its ingroup and outgroup members. And the preference of agent in group $A$ for its ingroup and outgroup members is $F_{\text {ina }}$ and $F_{\text {outa }}$, respectively. The preference of agent in group $B$ for its ingroup and outgroup members is $F_{\text {inb }}$ and $F_{\text {outb }}$, respectively.

If $F_{\text {in }}>F_{\text {out }}$, the agent will prefer ingroup members over outgroups (i.e., ingroup bias); if $F_{\text {out }}>F_{\text {in }}$, the agent will prefer outgroup members over ingroups, (i.e., ingroup derogation); and if $F_{\text {in }}=F_{\text {out }}$, the agent has no clear bias between the groups. Furthermore, there is another Identity Bit-string with only one bit in length and its value of 0 means that the agent belongs to the group $A$, and 1 mean that the agent belongs to the group $B$.

Moreover, when a female agent reaches the minimum reproductive age $R$, she will randomly select a male agent with the same group identity and age not less than $R$ to reproduce an offspring. The offspring's chromosomes are constructed by randomly crossing the chromosomes of parents as described in the sexual Penna model [22]. Mutations are introduced during reproducing process, with $M_{f}$ mutations on father's gametes, and $M_{m}$ mutations on mother's gametes.

\section{B. Disease Transmission}

Considering that the genes of pathogens are constantly changing in the course of evolution and in order to ensure that 
the pathogens have sufficient selection pressure on the agents. The mutation of pathogen Bit-string is set as following: Mutations occur in $D$ randomly selected positions of Bit-string with $P_{m u}$ probability each year, and if the mutation occurs in the pre-defined selective region, the content of the selected position is randomly switched with the content of another position in the pre-defined selective region. Otherwise, the value of the selected position goes from 0 to 1or from 1 to 0 . Due to the contacts between the agents and their habitat, such as through animals, water, feces, etc., pathogens can infect an agent with $P_{y}$ probability through the environment. And we calculate the possibility of the agent suffering from an infectious disease with the Hamming distance [26]. Such as, for pathogen $a$, we match the Health Bit-String with the corresponding gene on the Bit-string $i l l_{a}$, at the position of $i$, the Hamming distance of the agent with $z$ years old is $D_{a}(\mathrm{z})=$ $\sum_{i=1}^{z}|I a(i)-H(i)|$, Where $I a(i)$ is the gene of Bit-string $i l_{a}$. We set that the probability $P_{y}=\exp \left[-\partial * D_{a}(z) /\left(z-D_{a}(z)\right)\right]$, where $\partial>0, \partial=1 / F_{a}$, the smaller the value of $\partial$, the higher the load of pathogens in the environment and the greater the probability that the agent will contract an infectious disease; and the more consistent the genes between pathogen Bit-string and Health Bit-string, the smaller the value of $D_{a}(\mathrm{z})$, the higher the value of $P_{y}$. Moreover, when $\partial=D_{a}(z), P_{y}=0$. The same principle also applies to pathogen $b$. In addition, because of social cooperation and social exchange among the ingroup members, pathogens can also infect $N$ agents with $P_{p}$ probability through one sick agent. Furthermore, in our model, agents can be infected by a variety of infectious diseases, but they cannot repeatedly be infected with the same infectious pathogen (i.e., the infectious disease cannot be cured in this model). If an agent suffers from pathogen $a$, it will die with $P_{a}$ probability each year. As the same, agents who suffer from pathogen $b$ will die with $P_{b}$ probability each year.

Agents have the opportunity to join another social group each year [13, 14]. The probability of this opportunity is determined by the group attitudes of the agent and the residual resource value of the two regions. For example, for the agent belongs to group $A$, in each year, it has the probability of $Y_{a}=$ $\left(\left(\left(1-N_{t}(a)\right) / N A_{\max }\right)+F_{\text {in }}\right)-\left(\left(\left(1-N_{t}(b)\right) / N B_{\max }\right)+F_{\text {out }}\right)$, where $\left(1-N_{t}(a)\right) / N A_{\max }$ and $\left(1-N_{t}(b)\right) / N B_{\text {max }}$ represent the value of residual resources in lattice $A$ and lattice $B$. If $Y_{a}<0$, the outgroup (group $B$ ) has advantage and this agent has the intention to change the identity of group; if $Y_{a} \geq 0$, the ingroup (group $A$ ) has advantage and this agent hasn't intention to change the identity. However, the final group identity is determined by the agent's intention and the average degree of acceptance of the target group. For example, for the agent in group $A$, the probability $P_{c}$ of the agent changing the group's identity is $P_{c}=F_{\text {outa }} \times$ mean $F_{\text {outb }}$, where mean $F_{\text {outb }}$ is the mean preference of agent in group $B$ for outgroup.

\section{The Order of Events}

In each year, the order of events is as follows: (1) the mutation in pathogen gene; (2) the growing of agents; (3) the changing of identity; (4) infecting pathogen through environment; (5) spreading pathogen through sick agent; (6) reproduction of agent.

\section{Simulation Protocol}

In order to ensure that agent can survive on their habitat, and that the pathogen has sufficient selection pressure on the agents, the setting of Bit-string $i l l_{a}$ and $i l l_{b}$ was as follows: the first fifty genes were defined as the selective region and the values of twenty genes in the first fifty genes were randomly set to Fig. 1.

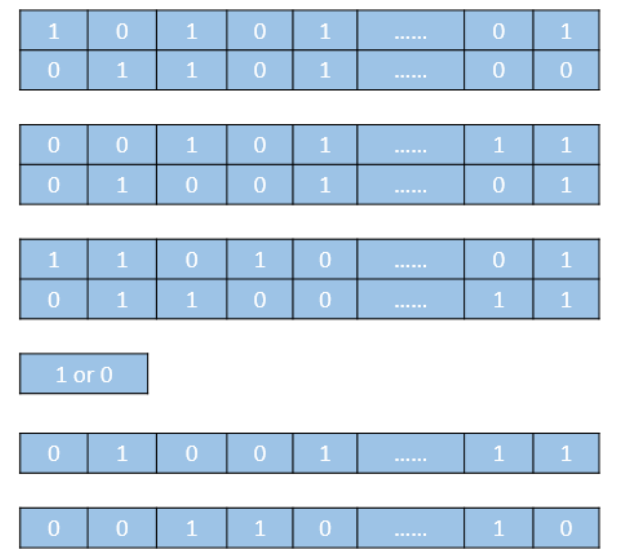

Heath Bit-string

Ingroup Bit-string

Outgroup Bit-string

Identity Bit-string

Pathogen $i l l_{a}$ Bit-string

Pathogen $i l_{b}$ Bit-string

Fig. 1. The first four Bit-strings represent the agents and the last two Bitstrings represent the pathogens.

To make the model reasonable and tractable, the setting of parameters was as following: $N_{a}=3750, N_{b}=3750, N A_{\max }=$ $35000, N B_{\text {max }}=35000, A_{\text {max }}=100, T=8, R=11, M_{f}=4, M_{m}$ = 4, $P_{m u}=0.001, D=1, P_{y}=0.8, P_{p}=0.1, P_{a}=0.15, P_{b}=0.15$, $N=4$. Simulations were carried out 20 times for a given set of parameters using different random seeds, and were stopped when the model had converged. The mean of the whole population in each time step was recorded as the data. We computed the average values of the data from last 10000 time steps.

\section{RESULTS}

The agents survived on the lattice with a very stable population size around 4060 under all parameter values. The results of the numerical simulations are shown in Fig. 2 and Fig. 3, where we plot the average stationary values of group attitudes (ingroup and outgroup) as a function of $F_{b}$. When the level of pathogen load factors $F_{a}$ was set to low $\left(F_{a}=0.001\right)$, and $F_{b}$ was not high $\left(F_{b}=0.5,0.8,1\right)$, with the increase of the value of $F_{b}$, the value of preference for ingroup members increased and the value of preference for outgroup members decreased in group $A$. It meant that agents belonged to group $A$ preferred ingroups because of the low prevalence rate of pathogen in group $A$. On the contrary, the value of preference for ingroup members decreased and the value of preference for outgroup members increased in group $B$. And when the level of pathogen load factors $F_{b}$ was set to high $\left(F_{b}=1.5,2,3\right)$, the value of preference for ingroup members increased and the value of preference for outgroup members decreased in group $A$. But the value of preference for ingroup members decreased and the value of preference for outgroup members increased in group $B$, and its value of preference for outgroups was over that value for ingroups when $F_{b}>1.5$. In summary, for the 
agents belonged to group $A$, there were strong preference for ingroups; and for the agents of group $B$, when the difference between the level of disease load in group $A$ and $B$ became bigger (the value of pathogen load difference was about 1.7), the group attitudes
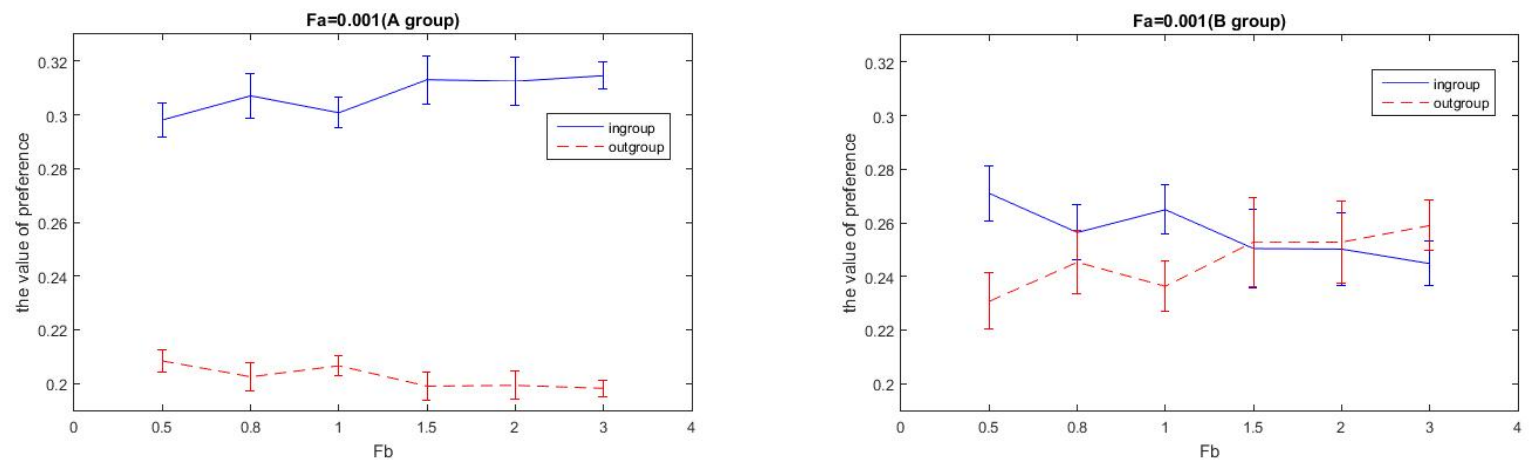

Fig. 2. Results for the relationship about pathogen load and the value of preference $\left(F_{a}=0.001\right)$. Error bars represent standard deviations.
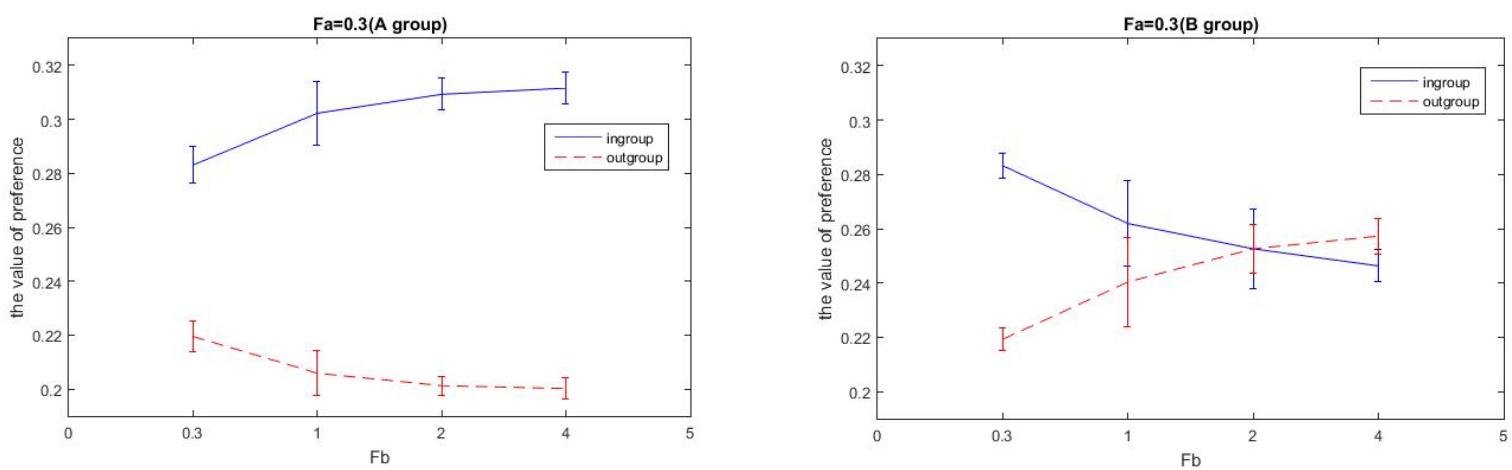

Fig. 3. Results for the relationship about pathogen load and the value of preference $\left(F_{a}=0.3\right)$. Error bars represent standard deviations.

As expected (see Fig. 3), the simulation showed similar results when the level of disease load factors $F_{a}$ was raised to a higher level of $\mathrm{F}_{\mathrm{a}}=0.3$, and the level of disease load factors $F_{b}$ was not very high $\left(F_{b}=0.3,1\right)$, the value of preference for ingroup members also decreased and the value of preference for outgroup members also increased in group $B$. When the level of $\mathrm{b}$ disease load factors $F_{b}$ was set to high $\left(F_{b}=2,4\right)$, for the agents of group $B$, this tendency was further enlarged, and the group attitudes were reversed that the ingroup bias was transformed into ingroup derogation. In addition, when the value of pathogen difference between ingroup and outgroup was about 1.7 , the agents in the group with higher disease prevalence would reverse their group attitudes in which the ingroup bias was transformed into ingroup derogation.

\section{DISCUSSION}

In present study, we utilized a Bit-string computational model to investigate the evolutionary origin of ingroup derogation phenomenon from the perspective of threat management. Results showed that, in the case of low disease prevalence of ingroup, ingroup members would prefer ingroups over outgroups; when the disease prevalence of ingroup increased, the value of preference for ingroup members decreased and the value of preference for outgroup members increased; when the value of pathogen load difference between were reversed that the ingroup bias was transformed into ingroup derogation (see Fig. 2). ingroup and outgroup was about 1.7, the agents in the group with higher disease prevalence would reverse their group attitudes in which the ingroup bias was transformed into ingroup derogation; and when the value of disease prevalence in ingroup further increased, the attitudes of ingroup derogation would be stronger. These results provide the direct evidence to our hypothesis that ingroup derogation can be evolved in the condition which ingroup members pose more threat of diseases than outgroup members.

Plenty of studies have simulated the evolution of ingroup bias within the framework of cooperation or altruism. They consistently reported the prevalence of infectious disease within the society's geographical region could influence the evolution of cooperation and the formation of social groups within social networks [24]. Our study also found that the ingroup bias can be evolved in the case of low prevalence of infectious disease within ingroups which supports the hypothesis that ingroup bias is an adaptation to the disease threat posed by outgroup members.

$\mathrm{Wu}$ et al. also tried to explain the aggressive behavior toward ingroup members by using a computational model and behavioral experiments [25]. They found that ingroup derogation could also be evolved if the chance of death incurred by intragroup conflicts was no less than 10\%. Further behavioral experiments on Chinese participants showed that 
the ingroup derogation mechanism responded to heuristic social category cues and it responded more strongly when participants subjectively felt more vulnerable to interpersonal aggression, or when there were contextual cues of aggression in the immediate environment. It suggests that the aggressive behavior and disease had interactively shaped the ingroup denigration mechanism. Future work should take this into consideration.

\section{REFERENCES}

[1] Jost, J. T., Pelham, B. W., Carvallo, M, “Non-conscious forms of system justication: Cognitive, affective, and behavioral preferences for higher status groups,”J. Exp. Soc. Psychol, vol. 38, pp. 586-602, 2002.

[2] Berhard, H., Fischbacher, U., and Fehr, E, "Parochial altru ism in humans," Nat, vol. 442, pp. 912-915, 2006.

[3] Allport, G, “The nature of prejudice,” J. Negro. Hist, vol. 52, pp. 390 393, 1954.

[4] Marques, J. M., Yzerbyt, V. Y., \& Leyens, J. P, "The 'Black Sheep Effect': Extremity of judgments towards ingroup members as function of group identification,” Eur. J. Soc. Psychol, vol. 18, pp.1-16, 1988.

[5] Ma-Kellams, C., Spencer-Rodgers, J., \& Peng, K, "I am against us? Unpacking cultural differences in ingroup favoritism via dialecticis m," Pers. Soc. Psychol. Bull, vol. 37, pp. 15-27, 2011.

[6] Zhao K, Wu Q, Shen XB, Xuan YM, Fu XL, "I undervalue you but I need you: the dissociation of attitude and memory toward in-group members,” PloS. One, vol. 7, e32932, 2012.

[7] Hewstone, M., and Ward, C, "Ethnocentrism and causal attribution in Southeast Asia," J. Pers. Soc. Psychol, vol. 48, pp. 614-623, 1985.

[8] Fincher. CL, Thornhill R, "Parasite-stress promotes in-group assortative sociality: the case of strong family ties and heightened religiosity," Behav. Brain. Sci, vol. 35, pp. 61-79, 2012.

[9] Schaller. M, Murray. DR, "Infectious disease and the evolution of crosscultural difference," in Evolution, culture, and the human mind, vol. III, Schaller. M, Norenzayan. A, Heine. SJ, Yamagishi. T, \& Kameda. T ,Eds. New York: Psychol. Press, 2010, pp. 243-256.

[10] Fincher C, Thornhill R. Assortative sociality, "limited dispersal, infectious disease and the genesis of the global pattern of religion diversity,” Proc. Biol. Sci, vol. 275, pp. 2587-2594, 2008.
[11] Fincher C, Thornhill R, "A parasite-driven wedge: infectious diseases may explain language and other biodiversity,” Oikos, vol. 117, pp. 1289-1297, 2008.

[12] Van Vugt M, Park JH, "Guns, germs, and sex: how evolution shaped our intergroup psychology,” Soc. Personal. Psychol. Compass, vol. 3, pp. 927-938, 2009.

[13] Schaller M, Neuberg SL, "Danger, disease, and the nature of prejudice(s),” Adv. Exp. Soc. Psychol, vol. 46, pp. 1-54, December 2012.

[14] Wu, Q., Tan, C., Wang, B., \& Zhou, P, "Behavioral immune system and ingroup derogation: the effects of infectious diseases on ingroup derogation attitudes," PloS. One, vol. 10, e0122794, 2015.

[15] Fincher, C. L., Thornhill, R., Murray, D. R., \& Schaller, M, "Pathogen prevalence predicts human cross-cultural variability in individualism/ collectivism,” Proc. Biol. Soc, vol. 275, pp. 1279-1285, 2008.

[16] Levinson, S. C., \& Gray, R. D, "Tools from evolutionary biology shed new light on the diversification of languages,” Trends. Cogn. Sci, vol. 16, pp. 167-173, 2012.

[17] Stauffer, D, “The Penna model of biological aging," Bioinform. Biol. Insights, vol. 1, pp. 91-100, 2007.

[18] Sousa, A. O, "Sympatric speciation in an age-structured population living on a lattice,” Eur. Phys. J. B, vol. 39, pp. 521-525, 2004.

[19] He, M. F., Pan, Q. H., and Yu, B. L, "Modeling epidemic based on Penna model,” Int. J. Mod. Phys. Vol. 16, pp. 799-805, 2005.

[20] Pan, Q. H., Yu, B. L., and He, M. F, "Evolutionary model with intelligence and knowledge,” Eur. Phys. J. B, vol. 48, pp. 575-581, 2005.

[21] Fincher, C. L., \& Thornhill, R, "Parasite-stress promotes in-group assortative sociality: The cases of strong family ties and heightened religiosity,” Behav. Brain. Sci, vol. 35, pp. 61-79, 2012.

[22] Sá Martins, J. S., and Stauffer, D, "Justification of sexual reproduction by modified Penna model of ageing," Physica. A. vol. 294, pp. 191 194, 2001.

[23] Thornhill, R., Fincher, C. L., and Aran, D, "Parasites, democratization, and the liberalization of values across contemporary countries," Biol. Rev. Camb. Philos. Soc, vol. 84, pp. 113-131, 2009.

[24] Brown, G. D. A, Fincher, C. L., \& Walasek, L, “Personality, Parasites, Political Attitudes, and Cooperation: A Model of How Infection Prevalence Influences Openness and Social Group Formation,” Top. Cogn. Sci, vol. 8, pp. 98-117, 2016.

[25] Wu, Q., Liu, Chen., Li., Li., \& Zhou, P, “A Price Paid for Our Internal Strife: Escalated Intragroup Aggression and the Evolution of Ingroup Derogation,” Front. Psychol, vol. 7, 1453, 2016. 